ORIGINAL PAPER

\title{
Peritoneal Fluid Stimulates NeOplastic transformation OF NORMAL HEK 293 CELLS BY HIGH EXPRESSION OF PLURIPOTENT GENES
}

\author{
Ilona Szabeowska-Gadomska ${ }^{1}$, Magdalena Ducher ${ }^{1}$, Magdalena Orzechowska ${ }^{1}$, \\ Joanna Bogusławska-Duch ${ }^{1}$, Magdalena Kowalska ${ }^{2}$, Helena Poławska ${ }^{1}$, Maciej Maeecki ${ }^{1}$
}

${ }^{1}$ Department of Applied Pharmacy, Medical University of Warsaw, Poland
${ }^{2}$ Department of Gynecology Oncology, Memorial Cancer Centre and Institute of Oncology, Warsaw, Poland

Gynecological cancers constitute a serious problem in the world. Their advanced stages are often characterized by the accumulation of ascites, which leads to spreading of cancer cells outside their primary focus. Despite progress in the treatment, prognoses are still not satisfactory. The main causes of these failures are chemoresistance, metastases and recurrences of the disease, which is influenced by, among others, the microenvironment of cancer cells. This study investigated the effect of the microenvironment, which create ascites derived from patients with ovarian and endometrial cancer to non-gynecological HEK 293 cells. The effect of the gynecological cancer microenvironment on HEK 293 cells behaviour was analysed using RT-PCR, qRT-PCR, Western blotting and functional analysis (invasion assays, hanging drop) methods. Our results suggest that the key genes for the development of cancer can be regulated by epigenetic and hypoxiainducible factor in dependent manner. It was observed that in vitro microenvironment, which is created by cells originating from patients with gynecological cancer (ovarian cancer, endometrial cancer) is able to generate changes in HEK 293 cells by itself.

Key words: microenvironment, ascites, ovarian cancer, endometrial cancer, pluripotency.

\section{Introduction}

Gynecological cancers, are common diseases among women worldwide. Endometrial cancer and ovarian cancer (C54 and C56 respectively, according to the International Classification of Diseases - ICD-10) are the most frequently registered gynecological malignancies in Europe [1]. Both of them are associated with the production of ascites. This fluid is rich in proteins and cells, such as: cancer cells, lymphocytes and mesothelial cells. Ascites can be considered as microenvironmental affection for cancer cells [2]. Neoplastic cells are presented as single cells or cell aggregates, and are able to spread the cancer to other parts of the human body. Alteration in gene activation, for instance silencing of tumor suppressors or activation of oncogenes, may occur in the case of cancer cells [3]. Dysfunction of cell adhesion (metastasis) is characteristic for tumor cells and is regarded as a result of alternation in E-cadherin expression (encoded by $\mathrm{CDH} 1)$. Furthermore, E-cadherin plays an important role in epithelial - mesenchymal transition (EMT) [4]. Some of the previous studies demonstrated the ability of ascites to modulate the gene expression, which is associated with changes in the cellular behavior of ovarian cancer cell [5, 6]. However, 
the influence of ascites or medium derived from ascites on non-gynecological cells is still unknown.

Neoplastic transformation is mainly connected with the combination of many factors, including genetic, epigenetic and environmental, which translates to specific gene activation [7]. Mutation of genes and epigenetic alterations underline oncogenesis and cancer progression [8]. It shall be noted that the changes in gene expression can be regulated by the process of methylation of DNA and histone modification (i.e. methylation, acetylation). DNA methylation is carried out by DNA methyltransferases (DNMTs: DNMT1, DNMT3a and DNMT3b). The product of methyl group transfer to cytosine in DNA is 5 -methylcitosine. The process of de novo methylation is referred to as the reaction, where the methyl group binds to the new sequence, which was not methylated before. Methylation of $\mathrm{CPG}$ island is involved in transcriptional repression [9]. Transcriptional activity can be modulated by post-transcriptional modification of histone. Moreover, histone modifications (for example acetylation) cause the increase of available DNA for transcriptional factors. This is associated with the transcriptional activity of genes [10].

OCT4, SOX2, REX1 and NANOG are pluripotency - associated genes, which are active in undifferentiated cells $[11,12]$. Some of them were used in the reprogramming process of human fibroblast cells [13] and sarcoma cells [14] to pluripotent cells. Zhang and his co-workers [14] demonstrated that sarcoma cells can be reprogrammed into pluripotent ones. In addition, their concept assumes that cells obtained from aggressive cancer can be terminally differentiated into other type of cells with the loss of tumorigenic potential [14]. Moreover, the expression of OCT4 (key pluripotency marker) in epithelial ovarian cancer cells may play an important role in initiation and progression of the malignancy process $[15,16]$.

\section{Aim of the study}

The aim of this study was to investigate the effect of the gynecological cancer microenvironment (the acellular fraction of ascites derived from patients with ovarian cancer and endometrial cancer and medium after in vitro cell culture those ascites) on HEK 293 cells (gene expression profiles, invasive tests).

\section{Material and methods}

\section{Patient ethical statement}

Following the patient's consent and the approval of the Local Ethical Committee (Maria Sklodowska Curie Memorial Cancer Centre Warsaw, Poland No 26/2012), there were collected ascites from those suffering from gynecological cancer.

\section{Cell culture}

There were collected approximately $50 \mathrm{ml}$ of ascites from two patients with advanced stage of cancer.

Firstly, from a 72 years old woman (CA125 125 IU/ $\mathrm{ml}$ ), who was diagnosed with IV clinical stage, G3 histological stage and adenocarcinoma endometrial histological type of endometrial cancer (according to FIGO) - Sample 1. The patient received chemotherapy carboplatin (CBP) and cyclophosphamide (CTX).

Secondly, from a 73 years old woman (CA125 240 IU/ $\mathrm{ml}$ ), who suffered from IIIC stage, G3 histological stage of ovarian cancer (according to FIGO) - Sample 2. The patient received chemotherapy Taxol and carboplatin (CBP).

The liquids were centrifuged (Eppendorf 5810R, Hamburg, Germany 200 x g for 5 min.). Supernatant liquid was achieved in $-80^{\circ} \mathrm{C}$ and after that it was used in further experiments. Pellet of cells was resuspended in medium A - DMEM + GlutaMAX (Thermo Fisher Scientific, Waltham, MA USA), with 10\% bovine serum added, (Lonza, San Diego, CA USA), as well as Antibiotic Antimicotic Solution 1:100 (Sigma - Aldrich, St. Louis, MO USA) and was incubated at $37^{\circ} \mathrm{C}$ and $5 \% \mathrm{CO}_{2}$. Afterwards, Sample 1 and Sample 2 cells were selected and propagated in the same culture conditions. The number of both samples increased and, after passaging the culture medium (acellular medium), they were included in further experiments.

\section{The effect of cancer microenvironment on HEK 293 cells}

To investigate the role of the gynecological cancer microenvironment there was used HEK 293 (ATCC CRL-1573 Human Embryonic Kidney). For RNA isolation and protein extraction, the HEK 293 was cultured in $25 \mathrm{~cm}^{2}$ bottles at a density of $4 \times 10^{5} /$ bottle (Thermo Fisher Scientific, Waltham, MA USA). After $72 \mathrm{~h}$ of incubation, the medium was changed. New medium consists of $6 \mathrm{ml}$ medium $\mathrm{A}$, with an additional $2 \mathrm{ml}$ medium of cultured cells derived from ascites (acellular medium Sample 1 or Sample 2, filtrated medium - 0,22 $\mu \mathrm{m}$ Millipore Billerica, MA USA). After 3 days, the cells were used for RNA and protein extraction. The experiment was performed in triplicate.

\section{Invasion assays}

Cellular invasion was assayed by determining the ability of cells to invade the synthetic membrane, with $8 \mu \mathrm{m}$ pore coated with matrigel (BD Bio Coat Matrigel Invasion Chambers, BD Biosciences, San Jose, CA USA). The number of $4,4 \times 10^{5}$ HEK 293 cells was seeded to the insert. Lower chambers were filled with: 
a) filtered ascites, acquired directly (acellular fraction derived from Sample 1 and Sample 2),

b) filtrated medium (acellular fraction); cultures of cell derived from Sample 1 and Sample 2 (25\% of total volume).

As a control, we used medium $A$, and filtered medium from cultured OVCAR-3 cells (ATCC HTB-161 ovarian cancer cells - positive control). After $48 \mathrm{~h}$ and $72 \mathrm{~h}$ of incubation, all the migrated cells that passed through the membrane were stained with Hoechst 33258 (Sigma - Aldrich, St. Louis, MO USA) and photographed (fluorescence microscope Axio Vert. A1, Zen 2010 - software Zeiss, Jena, Germany).

\section{Hanging drop}

After trypsinization of monolayer HEK 293, cells were counted and then $4 \times 10^{4}$ cells were suspended in filtrated medium from in vitro cultured Sample 1 and Sample 2 cells (25\% of total volume) or filtrated ascites from patients (Sample 1 or Sample 2). As a control, we used medium A and medium from cultured OVCAR-3 cells (positive control). After pipetting, the cells were applied on the bottom of the petri dish. The bottom of the culture dish was filled with PBS (Sigma - Aldrich, St. Louis, MO USA), in order to mainly drops of each variant were inverted and put on the bottom of the culture dish. The cells were incubated in the conditions described above for $48 \mathrm{~h}$ and $72 \mathrm{~h}$, and then photographed (IB-100, Delta Optical, ScopeImage 9.0 - software Warsaw, Poland).

\section{Quantitative RT-PCR and RT-PCR}

Total RNA was isolated using TRIzol ${ }^{\circledR}$ Reagent (Thermo Fisher Scientific, Waltham, MA USA), according to the manufacturer's protocol. Total RNA was treated with DNA - free ${ }^{\mathrm{TM}}$ Kit (Thermo Fisher Scientific, Waltham, MA USA) in order to eliminate possible genomic DNA contamination. One $\mu \mathrm{g}$ of RNA was reverse-transcribed with High Capacity RNA-tocDNA Kit (Thermo Fisher Scientific, Waltham, MA USA) according to the manufacturer's instruction.

Polymerase Chain Reaction was performed in BIORAD T100 ${ }^{\mathrm{TM}}$ Thermal Cycler (Hercules, CA USA). PCR products were separated into $1,5 \%$ agarose gels and visualized, using BIO-RAD ChemiDoc ${ }^{\text {тм }} \mathrm{MP}$ Imaging System (Hercules, CA USA).

Relative gene expression was analyzed by RealTime PCR using ViiA7 instrument (Thermo Fisher Scientific, ViiA ${ }^{\text {TM }} 7$ Software v.1.1 Waltham, MA USA). ACTB was used as an endogenous control. The following TaqMan probes were used in the RealTime PCR reaction: ACTB- Hs01060665_g1, MUC16-Hs01065189_m1, HIF1 $\alpha$-Hs00153153_m1, HIF $2 \alpha$ (EPAS1)- $\overline{\mathrm{H}} \mathrm{s} 01026149$ m1, NANOGHs04260366_g1, DNMT3A- $\mathrm{H} s 01027166$ m1, DNMT3B-Hs00171876_m1,HDAC1-Hs0060626료 g1,
HDAC2- Hs00231032_m1, CDH1-Hs01023894_m1 (Thermo Fisher Scientific, USA).

\section{Western blotting}

Cells were lysed in a lysis buffer (1\% NP40 in $20 \mathrm{mM}$ Tris -Sigma - Aldrich, St. Louis, MO USA, pH 7,4) with protease inhibitor cocktail (Sigma - Aldrich, St. Louis, MO USA). Protein concentrations of the samples were determined using a Bradford Reagent (Sigma - Aldrich, St. Louis, MO USA). The proteins $(20 \mu \mathrm{g}$ ) were separated by electrophoresis in $10 \%$ SDS polyacrylamide gels, and then transferred to PVDF membrane (BIO-RAD, Hercules, CA USA). Membranes were incubated with a $5 \%$ low fat milk/TBST (50 mM Tris, $150 \mathrm{mM} \mathrm{NaCl}, 0.1 \%$ Tween 20; Sigma - Aldrich, St. Louis, MO USA) for 1 hour, and then they were incubated overnight with anti-Nanog antibody (polyclonal, 1 : 500; Abcam, Cambridge, England, United Kingdom) or anti-HIF1 $\alpha$ antibody (monoclonal, 1 : 500; Abcam, Cambridge, England, United Kingdom) at $4^{\circ} \mathrm{C}$. After washing, the membrane was incubated with HRP-conjugated secondary antibodies (1 : 5000; Cell Signaling Technology, Boston, MA USA). The study was performed using an enhanced chemiluminescence method (17). The signal intensity was analyzed using ChemiDoc MP Imaging System (BIO-RAD, Hercules, CA USA) and normalized on the basis of $\beta$-Actin (1 : 1000; Sigma - Aldrich, St. Louis, MO USA) levels.

\section{Statistical analysis}

The statistical analysis was conducted using GraphPad Prism 6 computer software by GraphPad Software Inc. The results of the experiments were analysed by One-way ANOVA and Bonferroni's multiple comparisons test in order to determine the statistically significant differences between the control and the studied samples. The differences were considered statistically significant at the confidence level below 0,05 .

\section{Results}

\section{Pluripotency genes and telomerase reverse transcriptase activity}

The starting population of lines, which were derived from a woman with advanced stage of endometrial cancer and ovarian cancer (Sample 1 and Sample 2 respectively) and HEK 293 cells, untreated and treated acellular medium from cells Sample 1 and Sample 2, were subjected to a gene expression analysis by RT-PCR and a real-time RT-PCR. The results showed that HEK 293 cells, treated with acellular medium from Sample 1 and Sample 2 cell, expressed some of pluripotency genes, such as OCT4, SOX2, REX1 and NANOG. Only Sample 1 cells did 


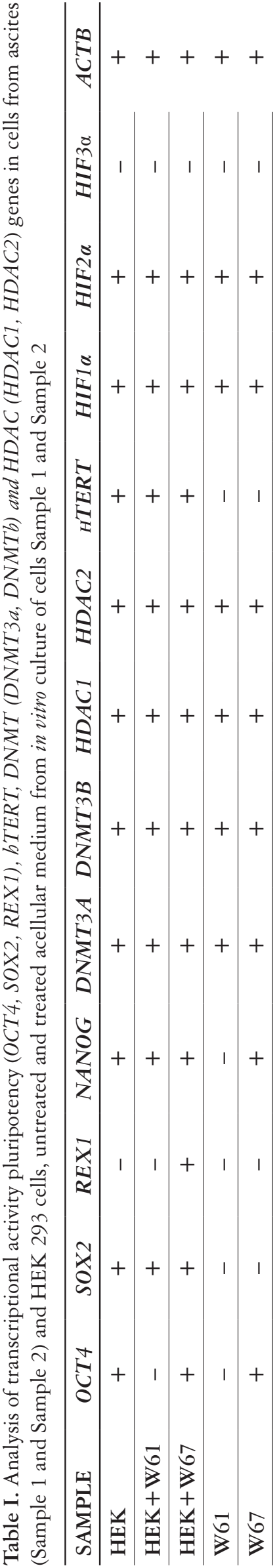

not express any pluripotency genes. However, HEK 293/Sample 1 expressed SOX2 (Table I). The results of a real-time PCR indicated that a sample HEK 293/ Sample 2 had a higher mRNA level of NANOG gene, compared to HEK 293 cells and Sample 2 cells. Moreover, the expression was higher even in comparison with HEK 293/Sample 1 (Fig. 1A).

HEK 293 cells cultured in an acellular medium derived from ascites and HEK 293 indicated the activity of a telomerase reverse transcriptase (bTERT) gene. However, cells, which were derived from patients and cultured in vitro did not express that gene (Table I).

\section{Hypoxia inducible factors}

All tested samples expressed HIF $1 \alpha$ and HIF $2 \alpha$ and none of them expressed HIF $3 \alpha$ (Table I). In relation to samples, no statistically significant difference was found in gene expression at mRNA level of HIF1a (Fig. 1B). The comparison made between the protein levels of HIF1 $\alpha$ in the tested samples indicated that Sample 2 cells demonstrated significantly higher level than Sample 1 ones (Fig. 1C). Moreover, HEK 293/Sample 2, indicated the highest mRNA level of HIF $2 \alpha$ and significantly higher level in comparison with HEK 293 cells (Fig. 1D). The ratio of HIF1 $\alpha$ / HIF $2 \alpha$ indicated that Sample 2 had significantly higher expression level of mRNA than HEK 293/ Sample 2 (Fig. 1E).

\section{CDH1 expression (encoding E-cadherin)}

HEK 293 cells, treated with the acellular medium from in vitro culture of Sample 1 cells (HEK 293/ Sample 1), represented significantly higher mRNA level of CDH1 gene compared to HEK 293 cells and Sample 1. Furthermore, the level was higher than in the case of Sample 2, HEK 293/Sample 1 vs. Sample 1 and HEK 293/Sample 2 vs. Sample 2 (Fig. 1F).

The analysis of each variant of experiments, which were carried out separately in probes (labelled as HEK 293/Sample 1 and HEK 293/Sample 2) showed that the expression of $C D H 1$ was the highest or nearly the highest, as compared to the other studied genes (Fig. 2A-D).

\section{DNA methyltransferases and histone deacetylases gene activity}

The RT-PCR analysis indicated that all tested variants expressed genes involved in methylation de novo (DNMT3a and DNMT3b), just as the I class of histone deacetylases (Table I).

There were statistically significant differences observed at the mRNA level of DNMT3a between HEK 293 cells treated with various media (Fig. 3A). The cells, which were incubated in the acellular medium taken from a patient suffering from ovarian 
A

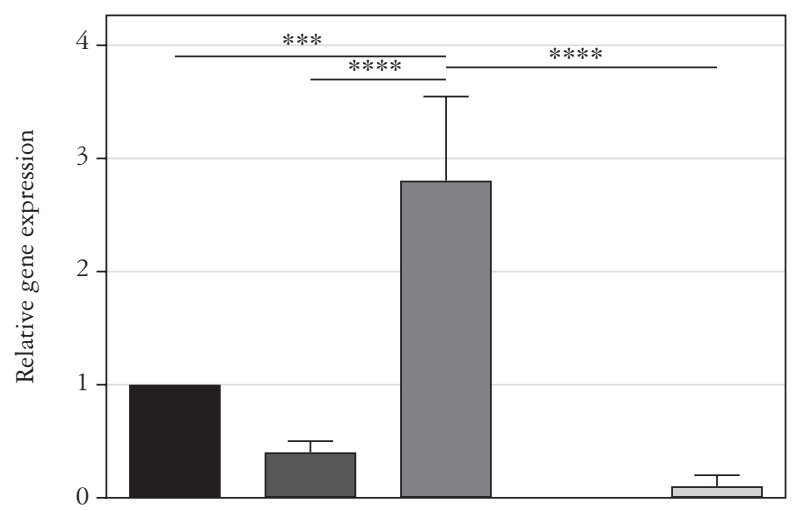

C

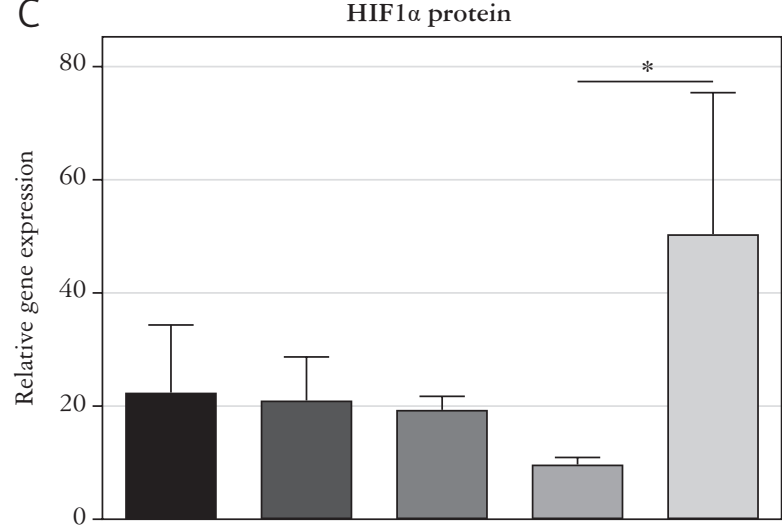

B

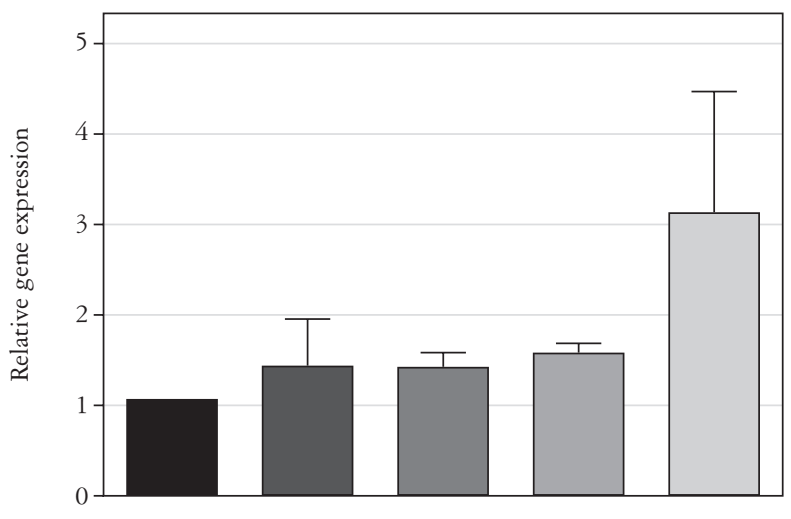

D

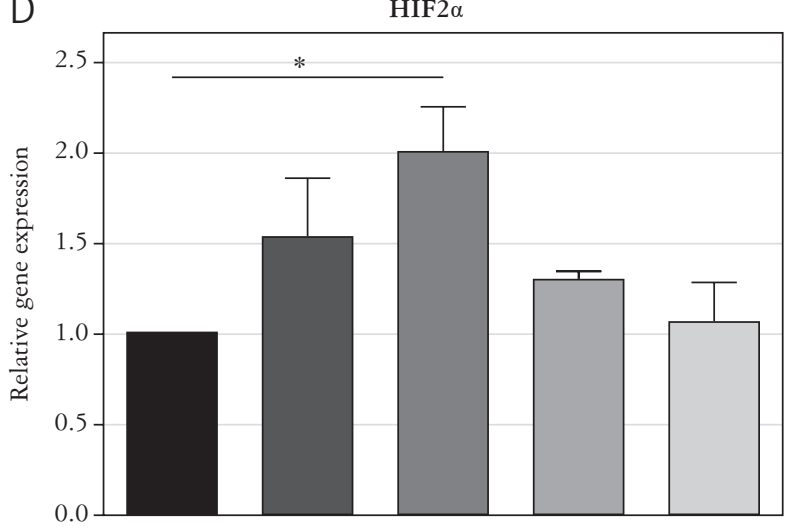

E

HIF $1 \alpha /$ HIF $2 \alpha$
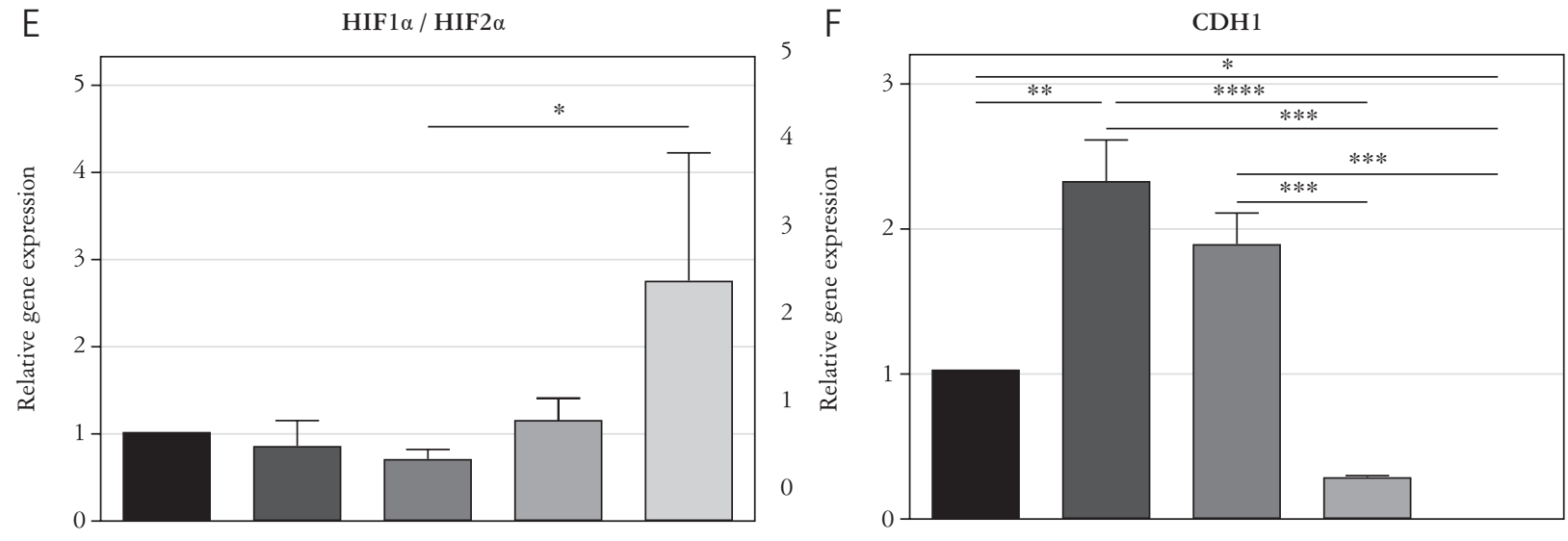

HEK 293

HEK 293/Sample 1

HEK 293/Sample 2

$\square$ Sample 1

$\square$ Sample 2

Fig. 1. The relative expression of NANOG gene (A) and HIF1 $\alpha$ gene (B) in cells from ascites (Sample 1 and Sample 2) and HEK 293 cells, untreated and treated acellular medium from cells Sample 1 and Sample 2. The relative protein level of HIF $1 \alpha$ is shown as relative to $\beta$-ACTIN in cells from ascites (Sample 1 and Sample 2) and HEK 293 cells, untreated and treated acellular medium from cells Sample 1 and Sample 2 (C). The relative expression of HIF2 $\alpha$ gene (D), HIF $1 \alpha /$ HIF $2 \alpha$ genes (E) and CDH1 gene (F) in cells from ascites (Sample 1 and Sample 2) and HEK 293 cells, untreated and treated acellular medium from cells Sample 1 and Sample 2. Data were presented as mean with SD of three independent experiments (Statistical analyses were done using One-way ANOVA and Bonferroni's multiple comparisons test, p $<0,05$ was considered as statistically significant one) 
A

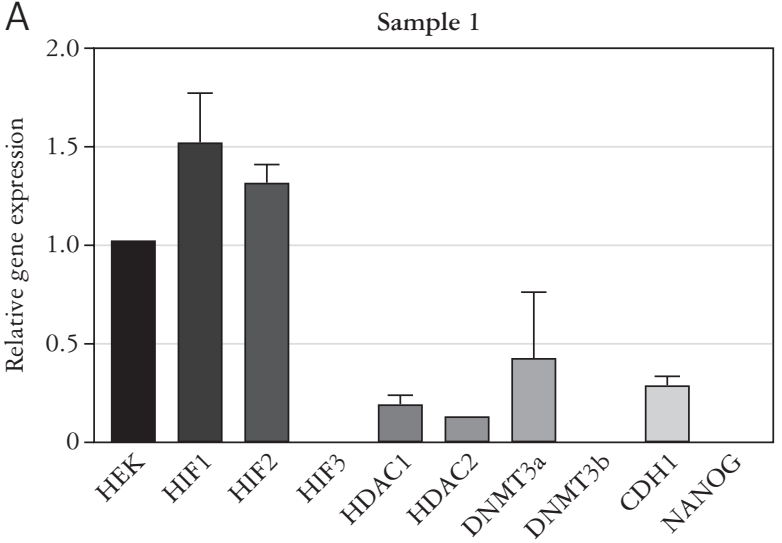

\begin{tabular}{|l|c|c|c|c|c|c|c|c|}
\hline \multicolumn{10}{|c|}{ Bonferroni's multiple comparison test, $\boldsymbol{\alpha}=0.05$} \\
\hline & HIF1 & HIF3 & HDAC1 & HDAC2 & DNMT3a & DNMT3b & CDH1 & NANOG \\
\hline HEK & $*$ & $* * * *$ & $* * * *$ & $* * * *$ & $* *$ & $* * * *$ & $* * *$ & $* * * *$ \\
\hline HIF1 & & $* * * *$ & $* * * *$ & $* * * *$ & $* * * *$ & $* * * *$ & $* * * *$ & $* * * *$ \\
\hline HIF2 & & $* * * *$ & $* * * *$ & $* * * *$ & $* * * *$ & $* * * *$ & $* * * *$ & $* * * *$ \\
\hline
\end{tabular}

C

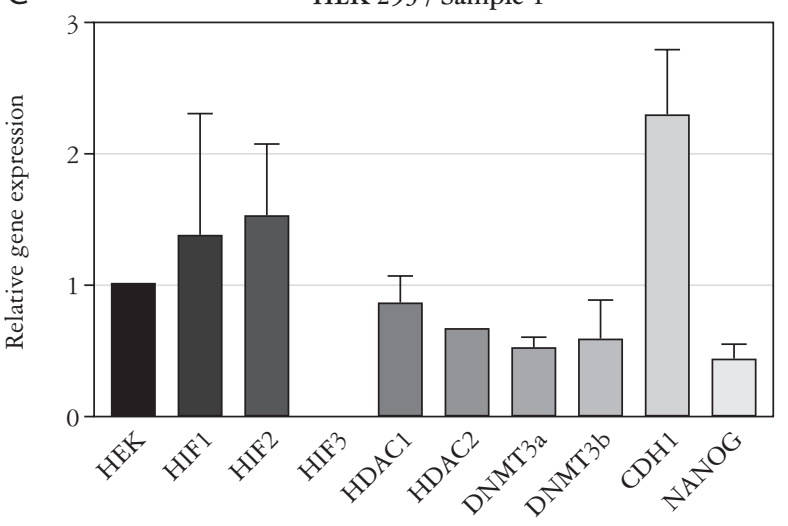

\begin{tabular}{|l|c|c|c|c|c|c|c|c|c|}
\hline \multicolumn{10}{|c|}{ Bonferroni's multiple comparison test, $\boldsymbol{\alpha}=0.05$} \\
\hline & HIF1 & HIF2 & HIF3 & HEK & HDAC1 & HDAC2 & DNMT3a & DNMT3b & NANOG \\
\hline HIF3 & $*$ & $* *$ & & & & & & & \\
\hline CDH1 & & & $* * * *$ & $*$ & $*$ & $* *$ & $* * *$ & $* *$ & $* * *$ \\
\hline
\end{tabular}
B

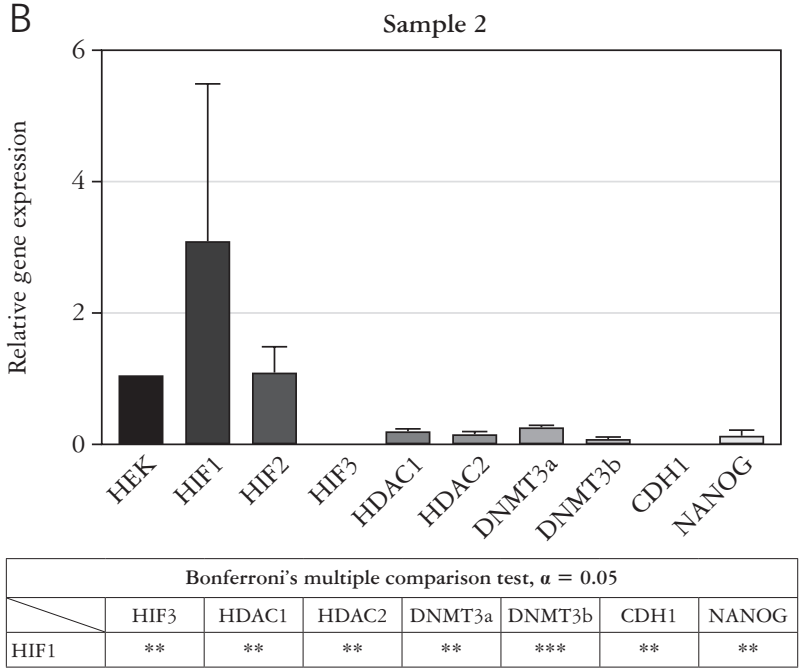

D

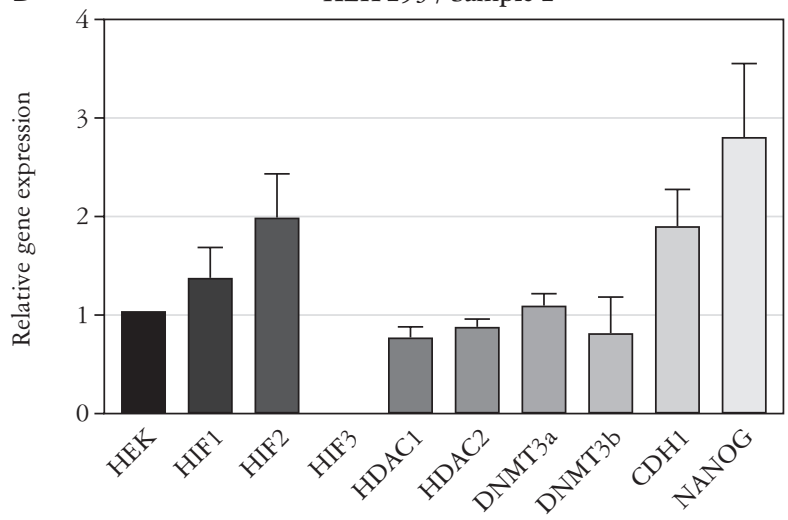

\begin{tabular}{|l|c|c|c|c|c|c|c|}
\hline \multicolumn{7}{|c|}{ Bonferroni's multiple comparison test, $\boldsymbol{\alpha}=0.05$} \\
\hline & HEK & HIF1 & HIF3 & HDAC1 & HDAC2 & DNMT3a & DNMT3b \\
\hline NANOG & $* * *$ & $* *$ & $* * * *$ & $* * * *$ & $* * * *$ & $* * *$ & $* * * *$ \\
\hline CDH1 & & & $* * * *$ & $*$ & $*$ & & $*$ \\
\hline HIF2 & & & $* * * *$ & $*$ & $*$ & & $*$ \\
\hline HIF1 & & & $* *$ & & & & \\
\hline
\end{tabular}

Fig. 2. The relative gene expression of HIFs, CDH1, NANOG, DNMTs and HDACs in cells from ascites: Sample 1 (A) and Sample 2 (B). HEK 293 cells and HEK 293 cells treated acellular medium from cells Sample 1 (HEK 293/Sample 1) (C) and Sample 2 (HEK 293/Sample 2) (D). Data were presented as mean with SD of three independent experiments (statistical analyses were done using One-way ANOVA and Bonferroni's multiple comparisons test, $\mathrm{p}<0,05$ was considered as statistically significant one)

cancer (HEK 293/Sample 2), indicated a higher level of mRNA than those incubated in the medium derived from patients with endometrial cancer (HEK 293/Sample 1). The mRNA levels of DNMT3a and DNMT3b were higher in case of control cells (HEK 293 cells), as compared to the cells derived from patients suffering from ovarian (Sample 2) and endometrial cancer (Sample 1) (Fig. 3A, B). The mRNA level of DNMT3b was significantly higher from HEK 293 treated acellular medium (HEK 293/ Sample 2), as compared to Sample 2 cells (Fig. 3B). The expression of $H D A C 1$ and $H D A C 2$ was the high- est in the case of control cells (HEK 293) (Fig. 3C, D). The results derived from Real-Time PCR demonstrated a significant difference between control cells and treated ones, in the expression of $H D A C 2$ genes. The cells treated with the acellular medium emanated from cells Sample 1 (HEK 293/ Sample 1) had the lower expression of $H D A C 2$ than control cells. In the case of cells derived from ascites of patients with gynecological cancer after in vitro culture (Sample 1, Sample 2), the expression of both genes HDAC1 and HDAC2 was lower, as compared to the control (HEK 293) (Fig. 3C, D). 
A

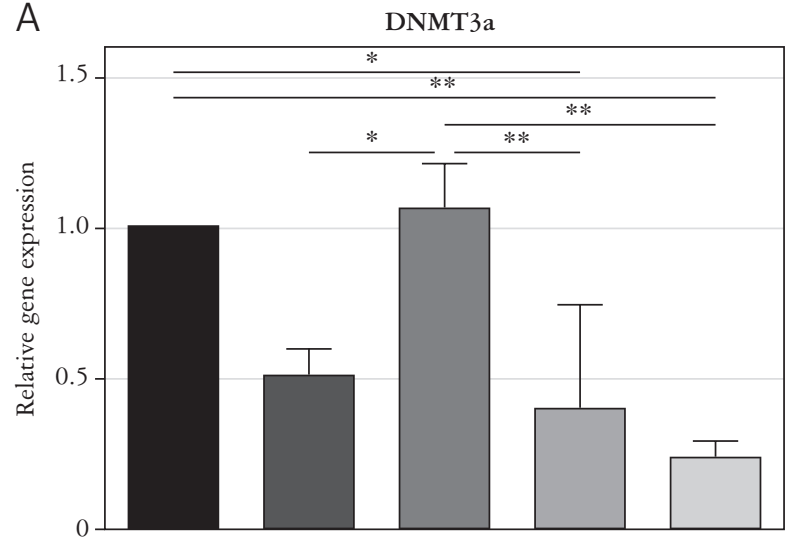

C

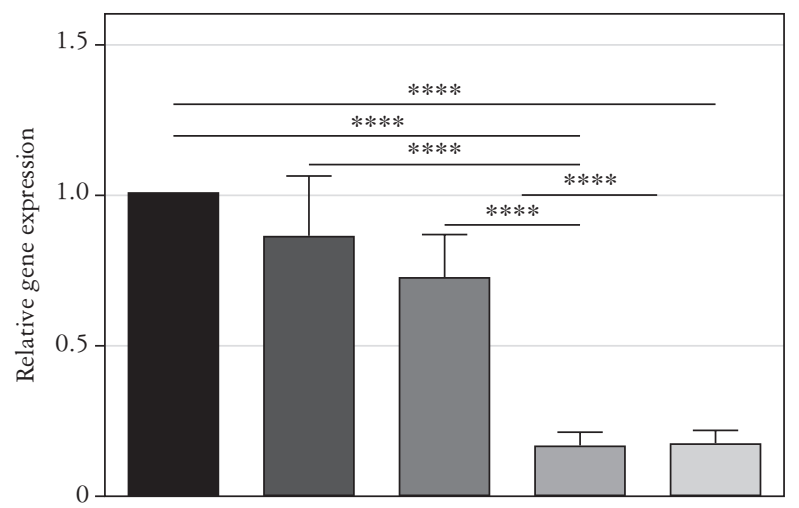

B

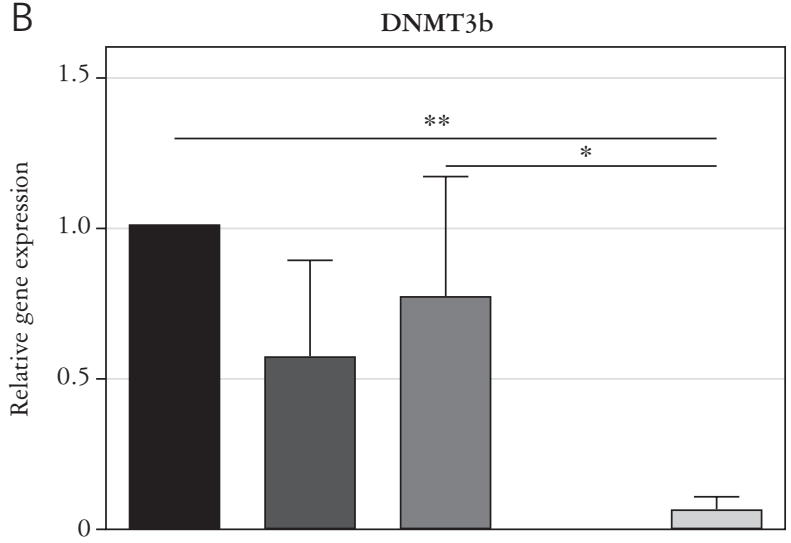

D

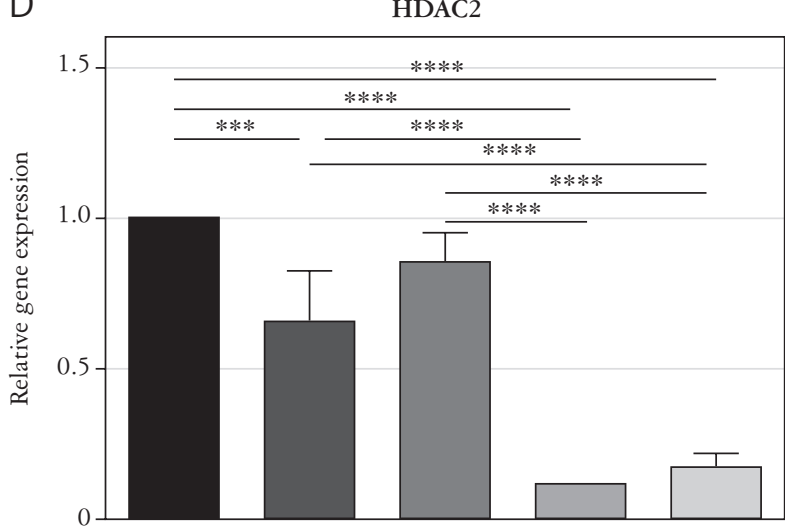

HEK 293

HEK 293/Sample 1

$\square$ HEK 293/Sample 2

$\square$ Sample 1

$\square$ Sample 2

Fig. 3. The relative expression of DNMT3a (A), DNMT3b (B), HDAC1 (C) and HDAC2 (D) genes in cells from ascites (Sample 1 and Sample 2) and HEK 293 cells, untreated and treated acellular medium from cells Sample 1 and Sample 2. Data were presented as mean with SD of three independent experiments (statistical analyses were done using One-way ANOVA and Bonferroni's multiple comparisons test, $\mathrm{p}<0,05$ was considered as statistically significant one)

\section{Summary of the results of gene expression}

The cells from ascites (Sample 1 and Sample 2) demonstrated the highest level of HIF1 $\alpha$, in comparison with other studied genes (Fig. 2A, B). However, the treated HEK 293 cells (HEK 293/Sample 1, HEK 293/Sample 2) presented a lower mRNA level of HIF1 $\alpha$ gene, as compared to HIF2 $\alpha$ (Fig. 2C, D).

The analysis of each variant of experiments, which were performed separately in probes showed that the expression of $C D H 1$ was the highest (HEK 293/ Sample 1) or nearly the highest (HEK 293/Sample 2) as compared to other studied genes in those variants. The sample HEK 293/Sample 2 indicated the highest expression of $N A N O G$ and also high expression of $C D H 1$ and $H I F 2 \alpha$ genes, as compared to other variants of experiments (Fig. 2). Cells treated with acellular medium (HEK 293/Sample 1 and HEK 293/Sample 2) were higher expression of HIF $1 \alpha, H I F 2 \alpha$ and $C D H 1$ genes as compared to control cells. Additionally, HEK 293 cells treated acellular medium of cells from patients suffering from ovarian cancer (HEK 293/Sample 2) were higher expression of NANOG and DNMT3a genes compare to HEK 293 cells treated medium of cells from patients suffering from endometrial cancer (HEK 293/Sample 1).

\section{Invasive assays}

The different kinds of acellular ascites meant that the HEK 293 cells formed an interesting pattern on the top of the membrane, which might give a visual image of the degradation of Matrigel components (Fig. 4A a-c). The variants with ascites (Sample 1 and Sample 2) created fields without cells (empty space) (Fig. 4A b-c). Such phenomenon was not observed in the case of the control variant (with the standard medium A, Fig. 4A a). Moreover, the fields that were created by cells treated with the fluid (received from patients suffering from ovarian cancer - Sample 2) (Fig. 4A c), were larger than those obtained 

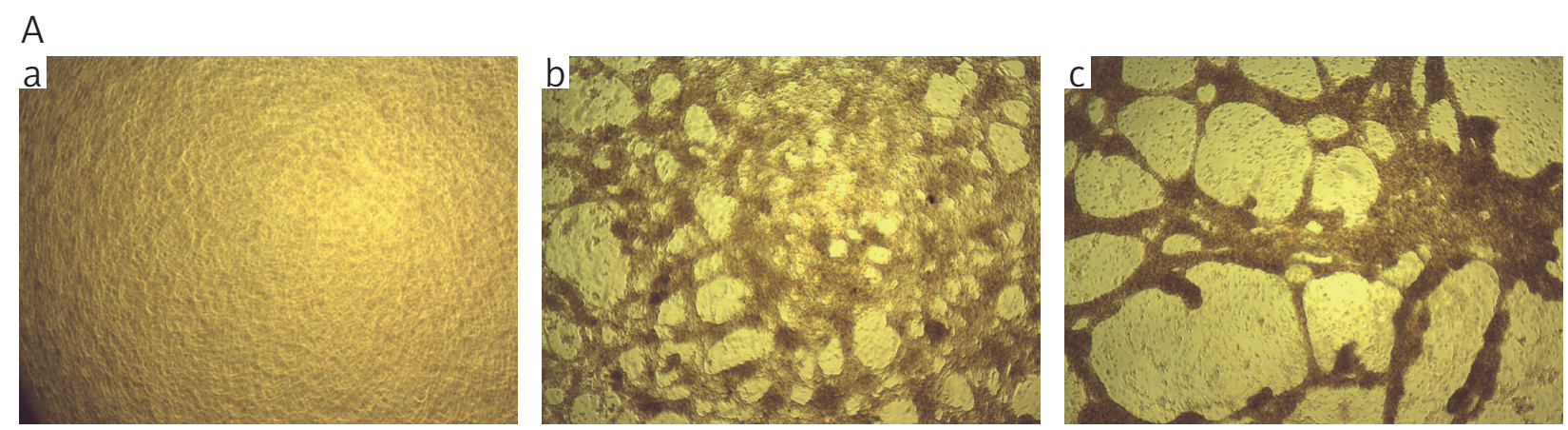

B
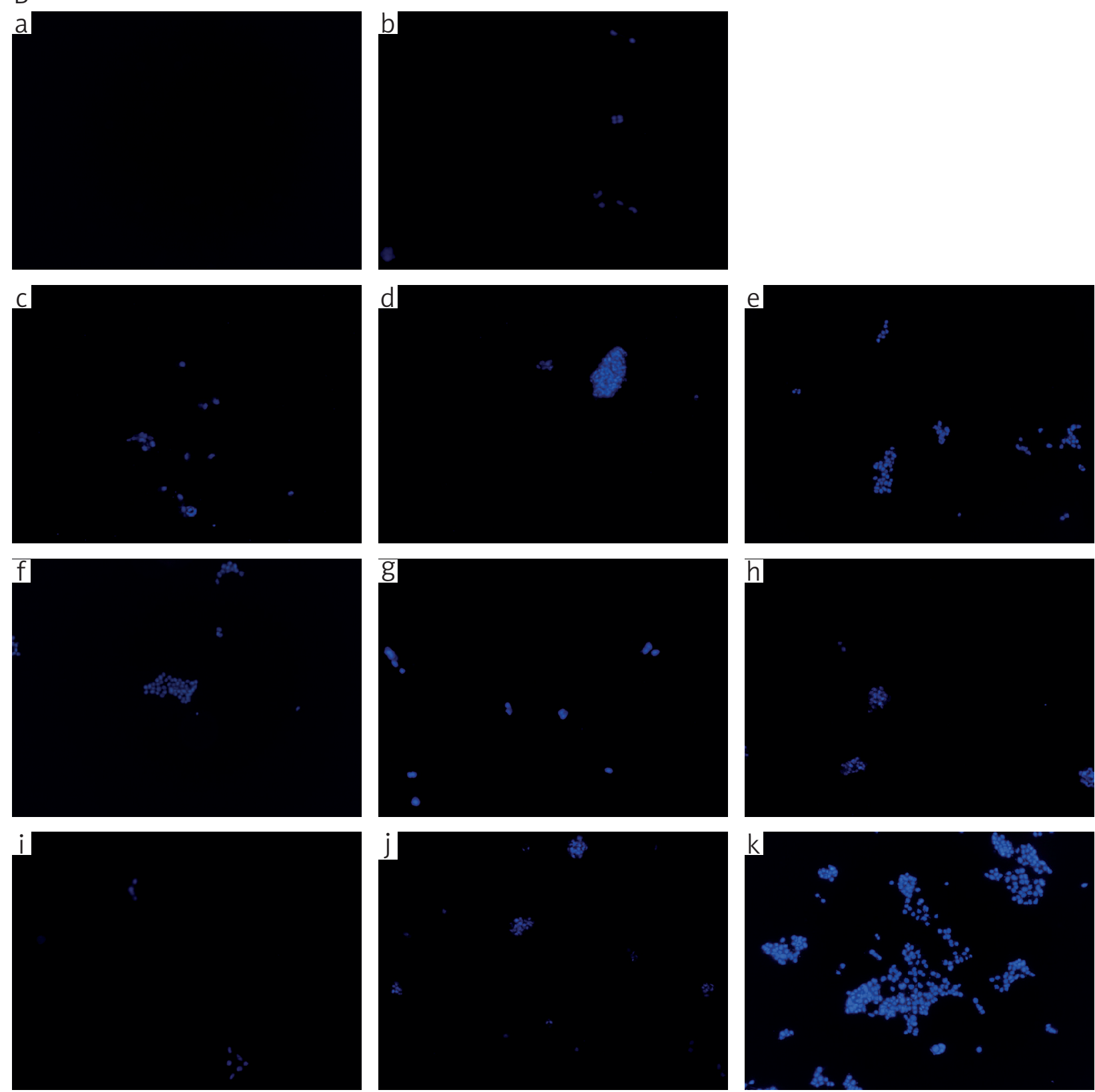

Fig. 4. A) The pattern of HEK 293 grow on the membrane surface. Influence of different attractant in low chamber: medium A (Control) - a, acellular ascites Sample 1 (the patient with endometrial cancer) - b, acellular ascites Sample 2 (the patient with ovarian cancer) - c on HEK 293 pattern growth on the top of membrane. B) Invasion assays. Hoechst staining after $48 \mathrm{~h}$ and $72 \mathrm{~h}$ of incubation of HEK 293 cells seeded on an insert, as a attractant in low chambers there were used: medium A (Control) - a, filtrated medium from OVCAR3 cells in vitro culture - b, acellular ascites from Sample 2 cells in vitro culture checked after $48 \mathrm{~h}-\mathrm{c}-\mathrm{j}$ and after $72 \mathrm{~h}-\mathrm{k}$. Control samples, samples of treated acellular medium from in vitro culture (Sample 1, Sample 2) and acellular ascites from sample Sample 1, did not cause invasion of HEK 293 cells (image the same as the control) 

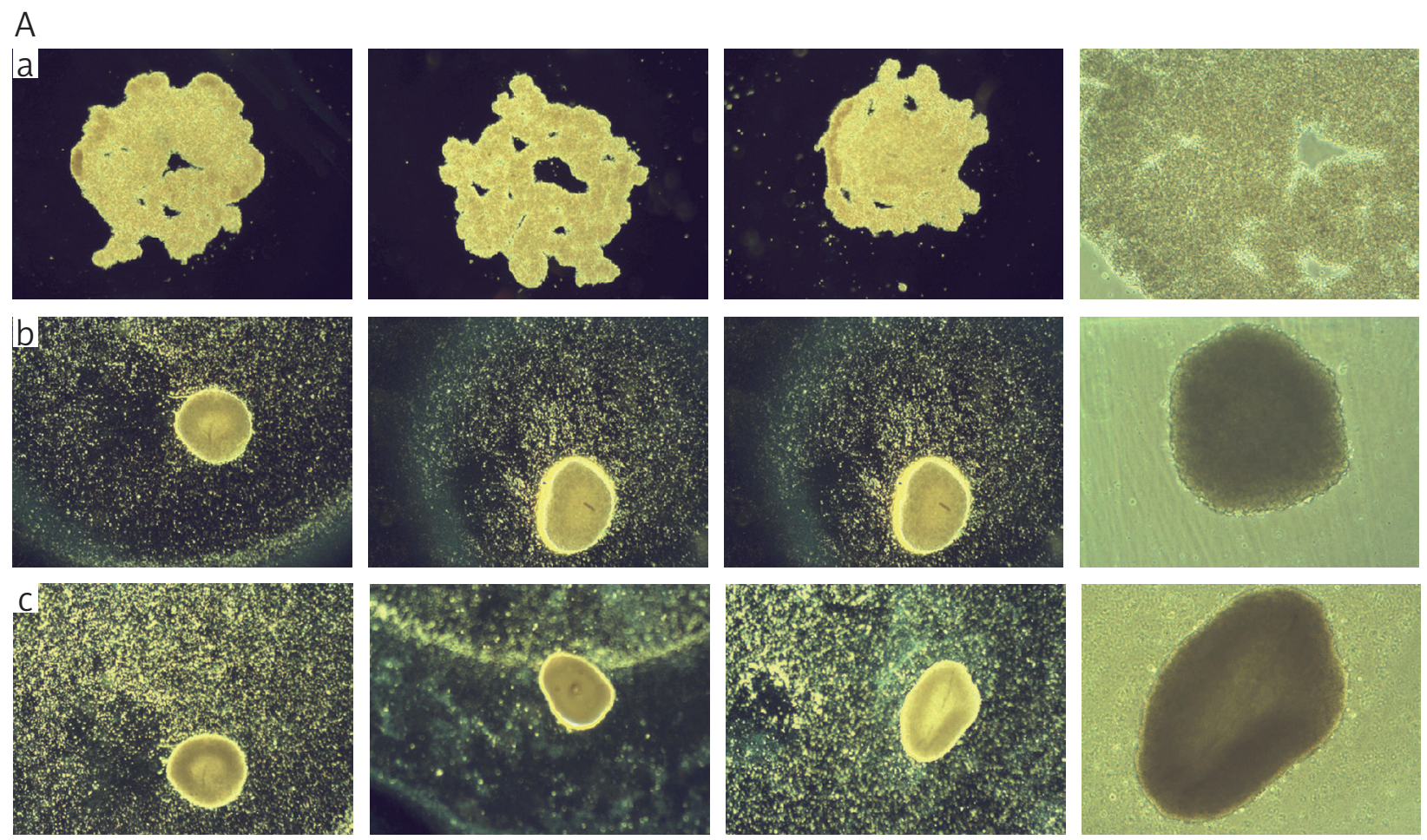

B
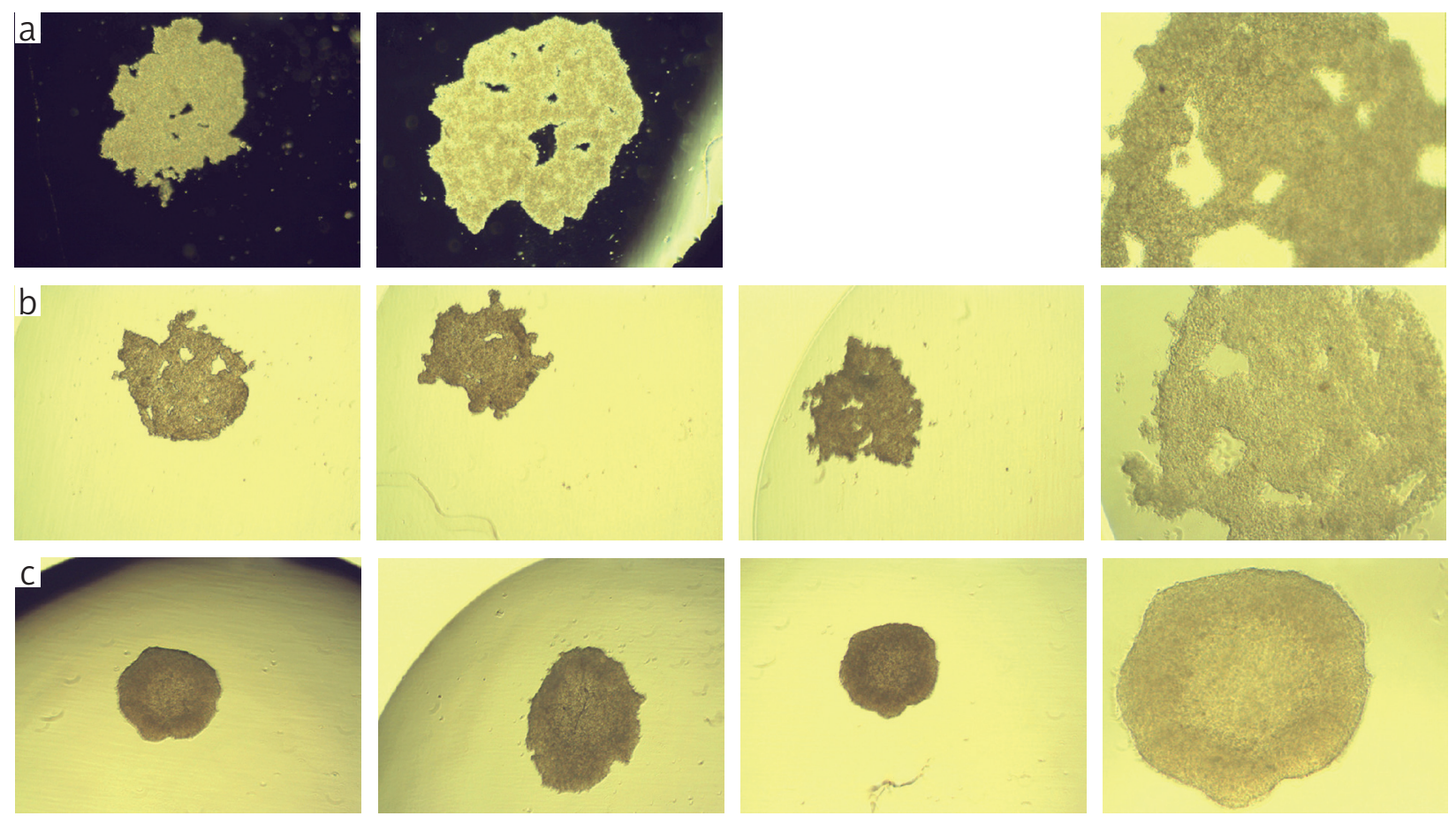

Fig. 5. A) Hanging drop culture. Spheroids of HEK 293 cells obtained after cultivation in: medium A (control) - a, acellular ascites from Sample 1cells - b, acellular ascites from Sample 2 cells - c. Magnification of the left side $40 \times$, right side $100 \times$. B) Hanging drop culture. Spheroids of HEK 293 cells obtained after cultivation in: medium A (control) - a, acellular medium from in vitro culture of Sample 1 cells $-\mathrm{b}$, acellular medium from in vitro culture of Sample 2 cells $-c$. Magnification of the left side $40 \times$, right side $100 \times$ 
from patients with endometrial cancer (Sample 1) (Fig. 4A b).

The results of invasive assays indicated that only filtrated ascites Sample 2 taken from patients were used as an attractant and a positive control with the medium received from in vitro culture of OVCAR 3 cells, which allowed observing the presence of cells in the bottom chamber (Fig. 4B a-k).

\section{Hanging drop}

The cultivation of HEK 293 cells in a different fluids generated various spheroids (Fig. 5A a-c, 5B a-c). Spheroids from the control medium gave larger and richer voids/empty spaces (Fig. 5A a, Fig. 5B a). The most regular and condensed shape represented spheroids cultured in acellular ascites Sample 1 (Fig. 5A b) and Sample 2 (Fig. 5A c), as well as the acellular medium from in vitro culture of Sample 2 cells (Fig. 5B c).

\section{Discussion}

Most ovarian cancers originate from the ovarian surface epithelium [18]. To improve the effectiveness of treatment of patients with ovarian cancer researchers should focus on understanding the molecular mechanism of invasiveness and metastasis [19]. The occurrence of metastasis causes a serious problem in relation to the cancer treatment. Metastasis of cancer cells into other organs from primary source of tumor usually means the progression of cancer disease and the bad prognosis [20]. Ovarian cancer metastasis is predominantly connected with the peritoneal cavity. Metastasis of adenocarcinoma is associated with migration and invasion of cancer cells [21]. On average, about $36 \%$ of cancer cells exist in ascites of patients with advanced stage of cancer [22]. Ovarian cancer cells can exist as single ones and MSC-multicellular spheroid. However, it is presumed that cells from spheroids are subject to the acquisition of more invasive properties [23].

The cells building the human body interact with ECM and neighbouring cells, which leads to the creation of a $3 \mathrm{D}$ structure. One model of in vitro 3D culture is multicellular spheroid (MSC). This model (presented by Holtfreter; Moscona and Moscona) shows more similarity, than traditional 2D culture systems (monolayer cells), to natural in vivo cells conditions [24].

The cell invasion assay allowed study the biological capabilities of cells. The co-occurrence of MMP2 and MMP9 entails the digestion of a MATRIGEL barrier of invasive chamber assays (the so-called mimetic natural basement membrane). The invasive cells move through the matrix towards the low chamber $[25,26]$. Our material was derived from patient's ascites with two frequently occurring gynecologi- cal types of cancer. Both women were at advanced stage of cancer disease. However, the results showed that the microenvironment that contained only fluid received from the patient suffering from ovarian cancer caused the migration of HEK 293 cells.

Furthermore, the HEK 293 cells as drops suspended in filtrated ascites Sample 2, created a compact spheroid structure. Sodek et al. previously reported about the positive correlation between the ability of invasion and the compact structure formation [23]. The results presented in this paper are in line with it. Moreover, the cells isolated from those ascites did not have the expression of E-cadherin, encoded by $C D H 1$ gene. This phenomenon correlated with the previous observation concerning the loss of expression of those molecules. Consequently, one observed the progression of malignancy and more invasive character of ovarian cancer cells [27, 28]. However, the presence and the role of E-cadherin in ovarian cancers cells are not clear and there are many antithetical hypotheses observed in this field. One of these paradigms assumes that E-cadherin plays a key role for a tumor suppressor [29]. Other hypothesis states that this diagnostic biomarker enhances the proliferation of tumor cells by the activation of MEK/ERK pathway [30]. Yet, the results of 60 cases showed that the negative staining of E-cadherin in patients suffering from ovarian cancer is associated with a shorter existence [31]. Simultaneously, there is astoundingly high level of E-cadherin observed in cells cultured in the medium derived from in vitro culture of Sample 1 and Sample 2 cells. It is assumed that a high presence of E-cadherin occurs at an early developmental stage of epithelial ovarian cancer [30]. This would mean that either HEK 293 cells affected by the environment of in vitro culture or those taken from patient with gynecological cancer, initiate neoplastic changes of ovarian cancer or, perhaps, the cells activate some protective mechanism defense against the induction of new neoplastic changes. Taking under consideration that activity of pluripotency genes may be involved in initiation of the malignancy process, the first hypothesis is supported by the overexpression of NANOG a and HIF2 $\alpha$ genes in HEK 293/Sample 2 cells, as compared to the control. Forristal and co-workers [32] presented the relation between HIF2 $\alpha$ and key pluripotency genes, including NANOG. Suppressing expression of HIF $2 \alpha$ caused a significant decrease in the expression of $N A N O G$ genes [32].

Recent reports also confirmed the observation of a positive influence of HIF $1 \alpha$ activation on the proteolytic activity, invasion and migration, as well as a simultaneous suppressing expression of E-cadherin $[33,34]$. The cells isolated from ascites Sample 2 pointed out a very high mRNA level of HIF1 $\alpha$, as compared to other tested genes. Additionally, a protein level confirmed the test of migration. Usually, 
the expression of HIF $\alpha$ proteins is associated with tumor grade and poor patient prognosis [35].

HDAC1 and HDAC2 belong to the I class of histone deacetylase family and, together with numerous protein co-repressors, are involved in the transcriptional repression and play multiple physiological roles $[36,37]$. In the study performed by Jin and co-workers [38], 15 of 18 ovarian cancer samples showed an increased expression of $H D A C 1$, while 12 of 18 ones pointed out an elevated expression of $H D A C 2$, regarding normal ovarian samples.

Hayashi et al. [39] reported that HDACs correlated to E-cadherin expression and proliferation marker (Ki67). Silencing in HDAC1 influenced a decreased proliferation of ovarian cancer cells, whereas silencing in HDAC3 caused the reduction of cell migration and an increased expression of E-cadherin. Another report presented the study on the influence of HDAC1, and co-repressors on the activity of E-cadherin promoter. The results showed that the transfection of HDAC1 modulated $40 \%$ of repression, while $80 \%$ were obtained by an additive effect of co-transfection HDAC1, Snail and $\mathrm{mSin} 3 \mathrm{~A}$ [40]. In our study, we observed that HEK 293 cells treated by the acellular medium (which was derived from filtrated ascites) had an increase in the expression of E-cadherin, whereas the expression of HDACs was lower, as compared to untreated cells. It confirms that an increase in one field entails a decrease in the other one. This reflects the fact that the expression of $\mathrm{CDH} 1$ gene (encoding E- cadherin) is regulated by HDAC1 and HDAC2.

\section{Conclusions}

The results presented in this article suggest that the gynecological microenvironment (ascites and acellular medium from in vitro culture) affects the expression of genes involved in pluripotency, metastasis, hypoxia inducible factors and genes encoding epigenetic enzymes (DNMTs and HDACs). Additionally, it alters the biological properties of cells. It was proved that cell fate can be modified by this environment. However, many issues still need to be resolved in order to apply this knowledge of cancer treatment in practice.

This work was partially supported by a grant from the National Centre for Research and Develoment (Strategmed 1/233624/4/NCBR/2014).

The authors declare no conflict of interest.

\section{References}

1. Ferlay J, Steliarova-Foucher E, Lortet-Tieulent J, et al. Cancer incidence and mortality patterns in Europe: estimates for 40 countries in 2012. Eur J Cancer 2013; 49: 1374-1403.

2. Matte I, Lane D, Bachvarov D, et al. Role of malignant ascites on human mesothelial cells and their gene expression profiles. BMC Cancer 2014; 14: 288.
3. Gerhauser C. Cancer chemoprevention and nutriepigenetics: state of the art and future challenges. Top Curr Chem 2013; 329: 73-132.

4. Yoshihara K, Tajima A, Komata D, et al. Gene expression profiling of advanced-stage serous ovarian cancers distinguishes novel subclasses and implicates ZEB2 in tumor progression and prognosis. Cancer Sci 2009; 100: 1421-1428.

5. Puiffe ML, Le Page C, Filali-Mouhim A, et al. Characterization of ovarian cancer ascites on cell invasion, proliferation, spheroid formation, and gene expression in an in vitro model of epithelial ovarian cancer. Neoplasia 2007; 9: 820-829.

6. Ahmed N, Riley C, Oliva K, et al. Ascites induces modulation of alpha6betal integrin and urokinase plasminogen activator receptor expression and associated functions in ovarian carcinoma. Br J Cancer 2005; 92: 1475-1485.

7. Herceg Z, Vaissière T. Epigenetic mechanisms and cancer: an interface between the environment and the genome. Epigenetics 2011; 6: 804-819.

8. Grønbaek K, Hother C, Jones PA. Epigenetic changes in cancer. APMIS 2007; 115: 1039-1059.

9. Bird AP, Wolffe AP. Methylation-induced repression - belts, braces, and chromatin. Cell 1999; 99: 451-454

10. Murphy SK. Targeting the epigenome in ovarian cancer. Future Oncol 2012; 8: 151-164.

11. Chambers I, Tomlinson SR. The transcriptional foundation of pluripotency. Development 2009; 136: 2311-2322.

12. Shi W, Wang H, Pan G, et al. Regulation of the pluripotency marker Rex-1 by Nanog and Sox2. J Biol Chem 2006; 281: 23319-23325.

13. Takahashi K, Tanabe K, Ohnuki M, et al. Induction of pluripotent stem cells from adult human fibroblasts by defined factors. Cell 2007; 131: 861-872.

14. Zhang X, Cruz FD, Terry M, et al. Terminal differentiation and loss of tumorigenicity of human cancers via pluripotency-based reprogramming. Oncogene 2013; 32: 2249-2260.

15. Peng S, Maihle NJ, Huang Y. Pluripotency factors Lin28 and Oct4 identify a sub-population of stem cell-like cells in ovarian cancer. Oncogene 2010; 29: 2153-2159.

16. Zhang Z, Zhu Y, Lai Y, et al. Follicle-stimulating hormone inhibits apoptosis in ovarian cancer cells by regulating the OCT4 stem cell signaling pathway. Int J Oncol 2013; 43: 1194-1204.

17. Haan C, Behrmann I. A cost effective non-commercial ECL-solution for Western blot detections yielding strong signals and low background. J Immunol Methods 2007; 318: 11-19.

18. Gardner MJ, Jones LM, Catterall JB, et al. Expression of cell adhesion molecules on ovarian tumour cell lines and mesothelial cells, in relation to ovarian cancer metastasis. Cancer Lett 1995; 91: 229-234.

19. Flate E, Stalvey JR. Motility of select ovarian cancer cell lines: effect of extra-cellular matrix proteins and the involvement of PAK2. Int J Oncol 2014; 45: 1401-1411.

20. Kramer N, Walzl A, Unger C, et al. In vitro cell migration and invasion assays. Mutat Res 2013; 752: 10-24.

21. Yamaguchi H, Wyckoff J, Condeelis J. Cell migration in tumors. Curr Opin Cell Biol 2005; 17: 559-564.

22. Gawrychowski K, Szewczyk G, Skopińska-Różewska E, et al. The angiogenic activity of ascites in the course of ovarian cancer as a marker of disease progression. Dis Markers 2014; 2014: 683757

23. Sodek KL, Ringuette MJ, Brown TJ. Compact spheroid formation by ovarian cancer cells is associated with contractile behavior and an invasive phenotype. Int J Cancer 2009; 124 : 2060-2070.

24. Lin RZ, Chang HY. Recent advances in three-dimensional multicellular spheroid culture for biomedical research. Biotechnol J 2008; 3: 1172-1184.

25. Soncin S, Lo Cicero V, Astori G, et al. A practical approach for the validation of sterility, endotoxin and potency testing 
of bone marrow mononucleated cells used in cardiac regeneration in compliance with good manufacturing practice. J Transl Med 2009; 7: 78.

26. Nieborowska-Skorska M, Hoser G, Rink L, et al. Id1 transcription inhibitor- matrix metalloproteinase 9 axis enhances invasiveness of the breakpoint cluster region/abelson tyrosine kinase-transformed leukemia cells. Cancer Res 2006; 66: 4108-4116.

27. Berx G, van Roy F. Involvement of members of the cadherin superfamily in cancer. Cold Spring Harb Perspec Biol 2009; 1: a003129.

28. Sawada K, Mitra AK, Radjabi AR, et al. Loss of E-Cadherin promotes ovarian cancer metastasis via alpha 5-integrin, which is a therapeutic target. Cancer Res 2008; 68: 2329-2339.

29. Lau MT, Klausen C, Leung PC. E-cadherin inhibits tumor cell growth by suppressing $\mathrm{PI} 3 \mathrm{~K} / \mathrm{Akt}$ signaling via $\beta$-catenin-Egr1-mediated PTEN expression. Oncogene 2011; 30: 2753-2766.

30. Dong LL, Liu L, Ma CH, et al. E- cadherin promotes proliferation of human ovarian cancer cells in vitro via activating MEK/ ERK pathway. Acta Pharmacol Sin 2012; 33: 817-822.

31. Daraï E, Scoazec JY, Walker-Combrouze F, et al. Expression of cadherins in benign, borderline, and malignant ovarian epithelial tumors: a clinicopathologic study of 60 cases. Hum Pathol 1997; 28: 922-928.

32. Forristal CE, Wright KL, Hanley NA, et al. Hypoxia inducible factors regulate pluripotency and proliferation in human embryonic stem cells cultured at reduced oxygen tensions. Reproduction 2010; 139: 85-97.

33. Jing SW, Wang YD, Chen LQ, et al. Hypoxia suppresses E-cadherin and enhances matrix metalloproteinase-2 expression favoring esophageal carcinoma migration and invasion via hypoxia inducible factor- 1 alpha activation. Dis Esophagus 2013; 26: 75-83.

34. Adamaki M, Georgountzou A, Moschovi M. Cancer and the cellular response to hypoxia. Pediatr Therapeut 2012; Suppl 1: S1.

35. Keith B, Simon MC. Hypoxia- inducible factors, stem cells, and cancer. Cell 2007; 129: 465-472.

36. Kelly RD, Cowley SM. The physiological roles of histone deacetylase (HDAC) 1 and 2: complex co-stars with multiple leading parts. Biochem Soc Trans 2013; 41: 741-749.

37. Dovey OM, Foster CT, Cowley SM. Histone deacetylase 1 (HDAC1), but not HDAC2, controls embryonic stem cell differentiation. Proc Natl Acad Sci USA 2010; 107: 8242- 8247.

38. Jin KL, Pak JH, Park JY, et al. Expression profile of histone deacetylases 1, 2 and 3 in ovarian cancer tissues. J Gynecol Oncol 2008; 19: 185-190.

39. Hayashi A, Horiuchi A, Kikuchi N, et al. Type- specific roles of histone deacetylase (HDAC) overexpression in ovarian carcinoma: HDAC1 enhances cell proliferation and HDAC3 stimulates cell migration with downregulation of E- cadherin. Int J Cancer 2010; 127: 1332-1346.

40. Peinado H, Ballestar E, Esteller M, et al. Snail mediates E-cadherin repression by the recruitment of the $\operatorname{Sin} 3 \mathrm{~A} /$ histone deacetylase 1(HDAC1)/HDAC2 complex. Mol Cell Biol 2004; 24: 306-319.

\section{Address for correspondence}

\section{Magdalena Ducher}

Department of Applied Pharmacy

Medical University of Warsaw

Banacha 1

02-097 Warsaw, Poland

e-mail: mducher@wum.edu.pl 